\title{
Measurement of the Bending Strength of Vapor-Liquid-Solid Grown Silicon Nanowires
}

\author{
Samuel Hoffmann, ${ }^{, \dagger}{ }^{\dagger}$ Ivo Utke, ${ }^{\dagger}$ Benedikt Moser, ${ }^{\dagger}$ Johann Michler, ${ }^{\dagger}$ \\ Silke H. Christiansen,,, Volker Schmidt,, Stephan Senz,, Peter Werner,, \\ Ulrich Gösele, ${ }^{\S}$ and Christophe Ballifl
}

\begin{abstract}
EMPA Materials Science and Technology, Feuerwerkerstrasse 39, 3602 Thun, Switzerland, Max Planck Institute of Microstructure Physics, Weinberg 2, 06120 Halle, Germany, Martin-Luther Universität Halle Wittenberg, Friedemann-Bach-Platz 6, 06099 Halle, Germany, and Institute of Microtechnology, University of Neuchâtel, A.-L. Breguet 2, 2000 Neuchâtel, Switzerland
\end{abstract}

Received November 11, 2005; Revised Manuscript Received January 30, 2006

\begin{abstract}
The fracture strength of silicon nanowires grown on a [111] silicon substrate by the vapor-liquid-solid process was measured. The nanowires, with diameters between 100 and $200 \mathrm{~nm}$ and a typical length of $2 \mu \mathrm{m}$, were subjected to bending tests using an atomic force microscopy setup inside a scanning electron microscope. The average strength calculated from the maximum nanowire deflection before fracture was around $12 \mathrm{GPa}$, which is $6 \%$ of the Young's modulus of silicon along the nanowire direction. This value is close to the theoretical fracture strength, which indicates that surface or volume defects, if present, play only a minor role in fracture initiation.
\end{abstract}

Nanowires (NWs) are of interdisciplinary interest to applications in the fields of biomedical sensing, nano- and optoelectronics and photovoltaics due to their electrical, optical, mechanical, and geometrical properties that may deviate substantially from bulk. ${ }^{1}$ To name some particularly exciting applications, the reader is referred to the following list: (i) high-frequency electromechanical resonators, ${ }^{2}$ (ii) high-aspect ratio tips for surface probe microscopy, ${ }^{3}$ (iii) sensor array for electrical detection of cancer markers, ${ }^{4}$ (iv) $\mathrm{Si} \mathrm{NW}$ arrays for photovoltaics, ${ }^{5}$ and (v) nanoscale light-emitting diodes. ${ }^{6}$ For all these applications the mechanical stability of the NWs is essential for their atomic scale manipulation, functionalization, or integration into device schemes.

Several methods were used in the past to access the mechanical properties of silicon NWs and nanobeams. An atomic force microscope (AFM) was used for bending tests of single crystal, micromachined silicon beams (from $1 \mathrm{~mm}$ down to $200 \mathrm{~nm}$ in width, beam axis oriented in [110] direction). No change in Young's modulus, but an increase in bending strength by a factor of up to 38 was observed from the millimeter down to the nanometer scale. ${ }^{7}$ AFM measurements were also done on silicon NWs (from 10 to

\footnotetext{
* Corresponding author. E-mail: samuel.hoffmann@empa.ch.

EMPA Materials Science and Technology.

$\doteqdot$ Martin-Luther Universität Halle Wittenberg.

$\S$ Max Planck Institute of Microstructure Physics.

"Institute of Microtechnology, University of Neuchâtel.
}

$100 \mathrm{~nm}$ in diameter, grown along the [111] direction) where a bending modulus of $186 \mathrm{GPa}$ (188 GPa in bulk) was measured. ${ }^{8}$

For NWs of gold $^{9}$ and silicon carbide, ${ }^{10}$ no dependence of the bending modulus on size was observed, but a fracture strength approaching the predicted theoretical maximum ${ }^{11}$ of $E / 10$ was measured for nanoscale objects, where $E$ is the Young's modulus. In contrast to these materials, a reduction of the bending modulus was observed for $\mathrm{ZnO}$ nanobelts in resonance experiments ${ }^{12}$ by a factor of 2 and for $\mathrm{ZnO}$ NWs in AFM measurements ${ }^{13}$ by a factor of about 4 compared to the bulk value. A recent paper reports bending strength values of vapor-liquid-solid (VLS) grown silicon NWs of around $500 \mathrm{MPa},{ }^{14}$ which is over a factor of 10 lower than what we found.

In this paper we present bending experiments on silicon NWs grown vertically and epitaxially on a [111] oriented silicon substrate. By direct observation and using elasticity theory as well as finite element (FE) simulations, the fracture strength of the NWs was determined. An estimation of the error is made and the VLS grown NWs are compared to micromachined silicon beams in terms of strength.

For the measurements an AFM was set up inside a scanning electron microscope (SEM). The AFM tip was used to bend a NW standing perpendicularly to the substrate until it fractured (Figure 1). A permanent deformation of the NW 


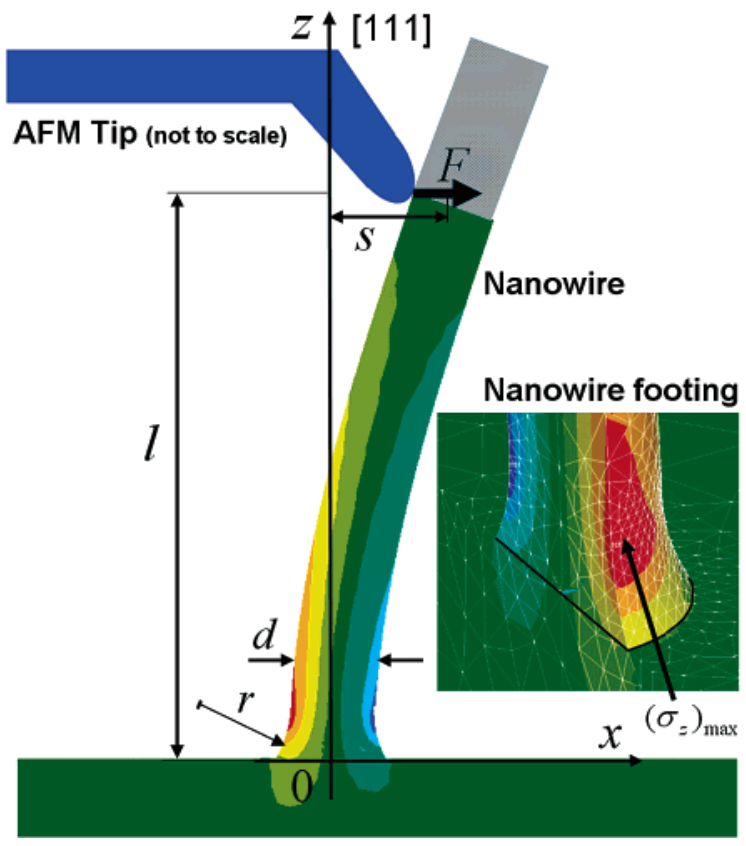

Figure 1. The NW stands perpendicular on the silicon (111) substrate. On the free end, an AFM tip is used to deflect the NW until it breaks. From the shape of the NW just before fracture, the maximum tension is calculated by means of formula 4 and threedimensional FE simulations. The upper part of the NW above the tip-NW contact is stress free and does need to be taken into account in the analysis. Inset: A detail of a FE simulation shows that the maximum tensile stress occurs where the NW becomes thicker, above the sharp corner to the substrate.

was not observed, even strongly deflected NWs (deflection $s /$ length $l>0.3$ ) snapped back to their original position when released. This allowed us to calculate the maximum stress from the NW deflection just before fracture.

In elastic, isotropic beam theory, the stress $\sigma_{z}$ at a point $x$, $z$ of the NW is ${ }^{15}$

$$
\sigma_{z}=-\frac{F(l-z) x}{I}
$$

where $F$ is the applied force, $l$ the NW length, and $I$ the geometrical moment of inertia. According to this formula, the maximum tensile stress occurs where the NW is attached to the substrate at $z=0, x=-d / 2(d$ being the diameter of the NW)

$$
\left(\sigma_{z}\right)_{\max }=\frac{F l d}{2 I}
$$

The force $F$ can be read out from the deflection $s$ and the spring constant $k$ of the NW

$$
F=k s=3 \frac{E I}{l^{3}} s
$$

where $E$ is the Young's modulus. Combining eqs 2 and 3 yields the maximum stress induced in the NW

$$
\left(\sigma_{z}\right)_{\max }=\frac{3}{2} \frac{d}{l^{2}} E s
$$

which is independent of the moment of inertia and thus independent of the NW cross section.

The NW diameter can be measured within an error of about $10 \%$ and its length and deflection within an error of about $5 \%$. This induces for these statistical errors a scatter on $\left(\sigma_{z}\right)_{\max }$ of $15 \%$.

$$
\frac{\delta\left(\sigma_{z}\right)_{\max }}{\left(\sigma_{z}\right)_{\max }}=\left(\frac{\delta d^{2}}{d^{2}}+\frac{4 \delta l^{2}}{l^{2}}+\frac{\delta s^{2}}{s^{2}}\right)^{1 / 2}
$$

If, however, because of the SEM characteristic such as its electron beam diameter and the image contrast there is a systematic error on $l, d$, and $s$ of $5 \%$, then an error of up to $20 \%$ may arise.

$$
\frac{\delta\left(\sigma_{z}\right)_{\max }}{\left(\sigma_{z}\right)_{\max }}=\frac{\delta d}{d}+\frac{2 \delta l}{l}+\frac{\delta s}{s}
$$

Silicon NWs grown epitaxially via the VLS mechanism show a larger diameter at the base of the $\mathrm{NW}^{16}$ (inset Figure $3 a)$. To verify the validity of the simple beam formula (3) for large deflections, low length $l /$ width $d$ ratios, and our particular shape of the NW footing, three-dimensional, anisotropic FE simulations with the commercially available software ANSYS (Ansys, Inc., Canonsburg, USA) were performed. The shape of the NW footing is modeled by a circle section of radius $r=100 \mathrm{~nm}$. The simulations show that the stress is highest in the region where the NW becomes thicker (inset Figure 1). Figure 2 shows that for high length/ width ratios the FE calculations exhibit a higher maximum stress than the linear beam formula 4, whereas for low length/ width ratios the stress from FE calculations is lower. This differences increase with increasing deflection. The resulting discrepancies between beam formula and FE simulations turned out to be around $\pm 10 \%$ for the geometries of the NWs of interest in this study (encircled values), which ranged from 90 to $190 \mathrm{~nm}$ in diameter and from 500 to $2000 \mathrm{~nm}$ in length.

The NWs were grown by the VLS growth mechanism in an ultrahigh vacuum environment using gold as catalyst and diluted silane as precursor (partial pressure $10 \mathrm{~Pa}$ ). The growth temperature was set to $500{ }^{\circ} \mathrm{C}$. The resulting NWs had an average diameter $d$ of approximately $100 \mathrm{~nm}$ and an aspect ratio $/ / d$ greater than 10 . Details of the growth process are described elsewhere. ${ }^{17}$

The AFM tip (AdvanceTEC, $45 \mathrm{~N} / \mathrm{m}$, Nanosensors, Neuchâtel, Switzerland) was mounted on a piezoelectric slip-stick robot arm (MM3A, Kleindiek Nanotechnik, Reutlingen, Germany) with two rotational and one linear axis. The substrate with the NWs is mounted on a $x, y, z$ piezo stage (P-620.2CD and P-62.ZCL, Physik Instrumente (PI), Karlsruhe, Germany) with $50 \mu \mathrm{m}$ range and sub-nanometer resolution. The whole setup was mounted inside a SEM (Hitachi Science Systems, Japan, S-3600N) such that the NWs are at an angle of $60^{\circ}$ with the scanning electron beam. With the 


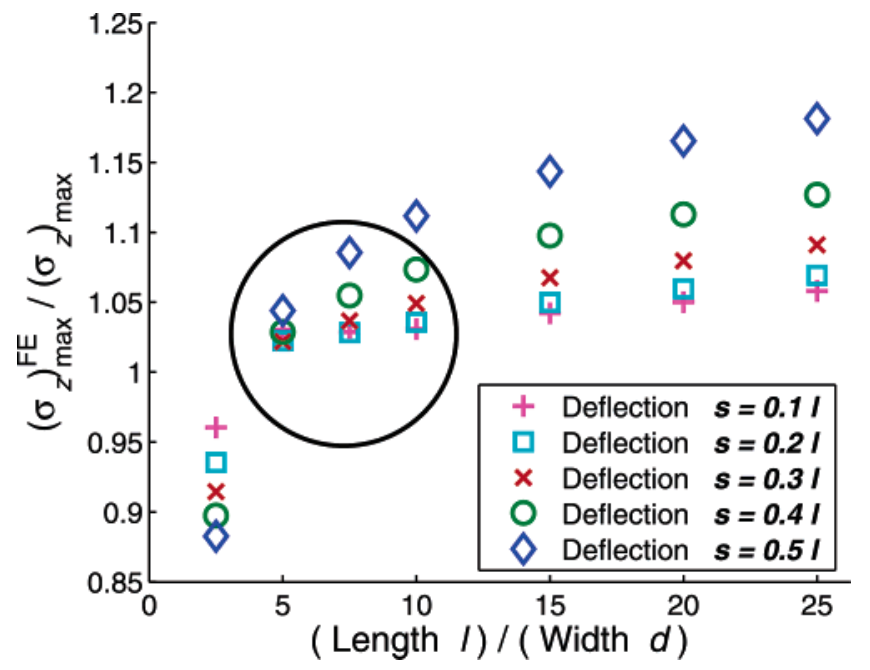

Figure 2. Three-dimensional FE calculations of the maximum stress $\left(\sigma_{z}\right)_{\max }^{\mathrm{FE}}$ compared to the linear beam formula result $\left(\sigma_{z}\right)_{\max }$ (eq 4). The circle indicates the length/width ratios of the NWs that were used in our experiments.

SEM table, the NW of interest was moved in the field of view. The coarse positioning of the AFM tip toward the sample was done with the robot arm, and the fine positioning as well as the bending of the NW was achieved by moving the sample with the $x, y, z$ piezo stage. The NWs were bent perpendicularly to the electron beam, so that the deflection $s$ could be read out directly from the SEM image. In the majority of cases, the AFM tip was not applied at the free end of the NW, but somewhere in the middle along its length (Figure 1). The bent section was then shorter than the NW, which gave the possibility of artificially shorten the length of the bent NW in order to get deflections that were not too large. The whole procedure was recorded in a video file (see Supporting Information). From the last image before fracture, the length $l$, diameter $d$, and deflection $s$ were extracted (Figure 3). With these parameters, the maximum stress has been calculated by eq 4 and by FE simulations.

The results from the experiments are listed in Table 1. As for silicon, no size dependence of the bending modulus was reported. We assumed the Young's modulus of bulk silicon [111], $E=188 \mathrm{GPa} .{ }^{18}$ The average fracture strength was 12 $\pm 3 \mathrm{GPa}$, which is around $6.3 \%$ of $E$. A few NWs fractured at the critical stress value of $E / 10$.

Although brittle materials have a probabilistic behavior in their mechanical properties, the scatter of the bending strength is expected to be smaller for such a small specimen.
Table 1. Measured Bending Strengths of the Silicon NWs Calculated by Means of the Simple Beam Formula $\left(\left(\sigma_{z}\right)_{\max }\right)$ and the FE Method $\left(\left(\sigma_{z}\right)_{\max }^{\mathrm{FE}}\right)$

\begin{tabular}{rrrrrrr}
\hline$d(\mathrm{~nm})$ & $l(\mathrm{~nm})$ & $s(\mathrm{~nm})$ & $l / d[1]$ & $\begin{array}{c}\left(\sigma_{z}\right)_{\max } \\
{[1 / E]}\end{array}$ & $\begin{array}{c}\left(\sigma_{z}\right)_{\max } \\
(\mathrm{GPa})\end{array}$ & $\begin{array}{c}\left(\sigma_{z}\right)_{\max }^{\mathrm{FE}} \\
(\mathrm{GPa})\end{array}$ \\
\hline 170 & 1650 & 370 & 9.7 & 0.035 & 6.5 & 7.0 \\
125 & 1500 & 480 & 12.0 & 0.040 & 7.5 & 8.4 \\
190 & 2000 & 580 & 10.5 & 0.041 & 7.8 & 8.8 \\
90 & 600 & 130 & 6.7 & 0.049 & 9.2 & 9.2 \\
115 & 1200 & 400 & 10.4 & 0.048 & 9.0 & 9.8 \\
120 & 850 & 230 & 7.1 & 0.057 & 10.8 & 11.1 \\
120 & 880 & 250 & 7.3 & 0.058 & 10.9 & 11.4 \\
125 & 750 & 190 & 6.0 & 0.063 & 11.9 & 11.9 \\
160 & 1050 & 300 & 6.6 & 0.065 & 12.3 & 12.7 \\
130 & 750 & 215 & 5.8 & 0.075 & 14.0 & 14.0 \\
150 & 760 & 210 & 5.1 & 0.082 & 15.4 & 15.1 \\
120 & 500 & 135 & 4.2 & 0.097 & 18.3 & 16.7 \\
100 & 570 & 210 & 5.7 & 0.097 & 18.2 & 18.1
\end{tabular}

Namazu et al. ${ }^{7}$ have found an average bending strength of $17.5 \pm 0.3 \mathrm{GPa}$ for micromachined silicon beams (along [111] direction) of $6 \mu \mathrm{m}$ length and around $250 \mathrm{~nm}$ in diameter. This corresponds to a scatter of $\pm 2 \%$, whereas for five of our NWs which have the same length of about 800 $\mathrm{nm}$, the scatter is $\pm 15 \%$. Aside from measurement inaccuracy, this could be due to the presence of surface or interfacial defects at the NW footing which lead to a stress concentration and initiate failure there. However, the NWs are essentially free of extended volume defects, and their influence is expected to be low as deduced from transmission electron microscopy, not shown here.

There does not seem to be a relation between strength and diameter. However, a correlation between strength and length has been observed. Shorter NWs tend to have a greater fracture strength than longer ones. If the tip-NW friction coefficient is high (adhesion due to electron beam induced contamination deposition), an additional tensile stress is induced in the NW, which is more important at large deflections. We assume that friction at the tip $-\mathrm{NW}$ contact may become important in long NWs because they tend to deflect strongly. This tensile stress is not accounted for in analytics and simulations and may explain the apparently weaker long NWs.

Recently, bending strengths of VLS grown NWs have been reported $^{14}$ that are a factor of more than 10 lower than the values we measured. The reported strengths, 300, 560, and $852 \mathrm{MPa}$, were read out from AFM force-deflection curves

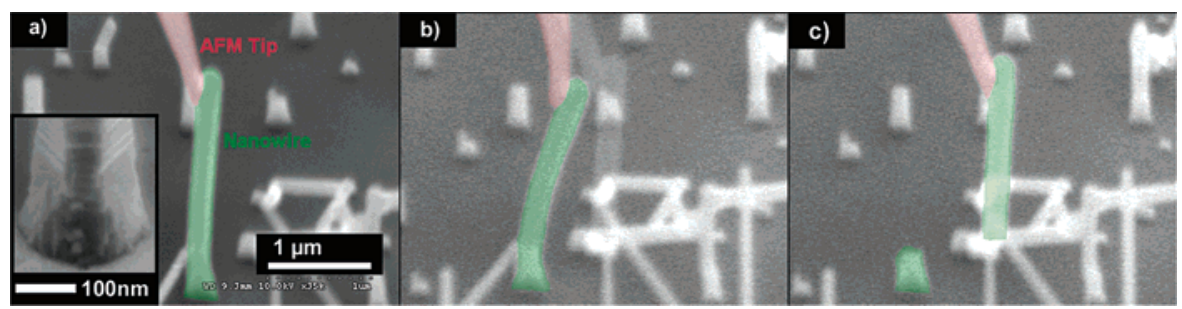

Figure 3. Tilt corrected SEM images from a bending experiment. (a) NW with AFM tip before deflection. Inset: The NW footing. (b) Image just before fracture. The AFM tip was bent, too. (c) Broken NW, the fracture occurred where the NW becomes thicker in good accordance with the FE simulations that indicate the maximum stress there. Mainly due to electron beam induced contamination deposition, the NW sticks to the AFM tip. 
on three NWs that had about the same diameter as our NWs but were much longer $(10 \mu \mathrm{m})$. Their results can only be explained in comparison to our results and results on micromachined silicon if defects played a dominant role in those experiments.

To conclude, the AFM inside the SEM can be used as a manipulator to bend and move nanoscale objects and perform quantitative measurements of their mechanical properties. FE calculations have shown that the linear beam formula can be used to calculate the maximum stress induced in the NW. On the basis of our results from bending silicon NWs, we estimate their fracture strength to be around $12 \mathrm{GPa}$, which is $6 \%$ of their Young's modulus. This is lower than what one would expect from the results of micromachined beams, ${ }^{7}$ but only a factor of 2 lower than the theoretical cohesive strength. Some NWs reached the theoretical cohesive strength, indicating that NWs can be grown virtually defect free by the VLS technique.

Acknowledgment. The authors thank NanoWorld AG Neuchâtel for providing the AFM tips, S. Fahlbusch for the helpful discussions on the AFM setup, and the Swiss National Science Foundation for financial support. S.H.C. thanks the German founding agency DIG for financial support under Contract Number CH 159/6-1.

Supporting Information Available: A video file of a bending experiment. This material is available free of charge via the Internet at http://pubs.acs.org.

\section{References}

(1) Law, M.; Goldberger, J.; Yang, P. Аnnu. Rev. Mater. Res. 2004, 34, 83.

(2) Husain, A.; Hone, J.; Postma, H. W. C.; Huang, X. M. H.; Drake, T.; Barbic, M.; Scherer, A.; Roukes, M. L. Appl. Phys. Lett. 2003, $83,1240$.

(3) Tay, A. B. H.; Thong, J. T. L. Appl. Phys. Lett. 2004, 84, 5207.

(4) Zheng, G.; Patolsky, F.; Cui, Y.; Wang, W. U.; Lieber, C. M. Nat. Biotechnol. 2005, 23, 1294.

(5) Peng, K.; Xu, Y.; Wu, Y.; Yan, Y.; Lee, S.-T.; Zhu, J. Small 2005, $1,1062$.

(6) Huang, Y.; Duan, X.; Lieber, C. M. Small 2005, 1, 142.

(7) Namazu, T.; Isono, Y.; Tanaka, T. J. Microelectromech. Syst. 2000 , 9, 450.

(8) San Paulo, A.; Bokor, J.; Howe, R. T.; He, R.; Yang, P.; Gao, D.; Carraro, C.; Maboudian, R. Appl. Phys. Lett. 2005, 87, 053111.

(9) Wu, B.; Heidelberg, A.; Boland, J. J. Nat. Mater. 2005, 4, 525.

(10) Wong, E. W.; Sheehan, P. E.; Lieber, C. M. Science 1997, 277, 1971.

(11) Dowling, N. E. Mechanical Behavior of Materials; Prentice Hall: Englewood Cliffs, NJ, 1999; ISBN 0-13-905720-X.

(12) Bai, X. D.; Gao, P. X.; Z. Wang, L.; E. Wang, G. Appl. Phys. Lett. 2003, 82, 4806.

(13) Song, J.; Wang, X.; Riedo, E.; Wang, Z. L. Nano Lett. 2005, 5, 1954.

(14) Tabib-Azar, M.; Nassirou, M.; Wang, R.; Sharma, S.; Kamins, T. I.; Saif Islam, M.; Williams, R. S. Appl. Phys. Lett. 2005, 87, 113102.

(15) Young, W. C. Roark's formulas for stress and strain; McGraw-Hill: New York, 1989; ISBN 0-07-072541-1.

(16) Schmidt, V.; Senz, S.; Gösele, U. Appl. Phys. A 2005, 80, 445.

(17) Schmidt, V.; Senz, S.; Gösele, U. Z. Metallkd. 2005, 96, 427.

(18) Wortman, J. J.; Evans, R. A. J. Appl. Phys. 1965, 36, 153.

NL052223Z 